\title{
Effect of Area Ratio on Base Pressure and Control Effectiveness
}

\author{
Fharukh Ahmed G. M., Sher Afghan Khan
}

\begin{abstract}
Reducing the base drag and increasing the base pressure from aerodynamic devices involving suddenly expanded flows is of vital importance due to the higher rate of drag associated with them. The experimental effort put into understanding the variation in base pressure using active control of suddenly expanded flows employing microjets is reported in this article. The effect of tiny jets and nozzle pressure ratio $(N P R)$, and length to diameter $(L / D)$ ratio on the percentage change in base pressure is investigated at supersonic Mach numbers at 1.7, 2.3, and 2.7, for area ratios of 2.56, 5.06, and 7.56. Apart from NPR, the $L / D$ ratio has an influential role in percentage change in base pressure at different Mach numbers. An improvement of up to $360 \%$ in base pressure is obtained with the use of sonic micro jets at a particular Mach number and $L / D$ ratio.
\end{abstract}

Keywords-Active control, microjets, Mach number, expanded flows, area ratio.

\section{INTRODUCTION}

In the area of ballistics, researchers have always been concerned about the issue related to external compressible flow in which the sudden expansion around the tail of projectiles and association of it with base pressure. In these flow areas, the base drag is around 10 percent of the drag caused by skin friction during the sub-sonic flow. Various techniques exist to reduce this base drag such as base burning, boattail, and base bleed. Moreover, the test data of most ballistics presented over the years reveals that the flight Mach number is an essential factor affecting base pressure [1]-[3]. In an internal combustion engine, the exhaust port has a similar flow condition where the jet of hot exhaust gases flows out through the exhaust value. The flow expansion is inward rather than outward in case of flow around the base of a missile in flight or blunt edged projectile, can be considered as another application of suddenly expanded flows [2]-[5].

The phenomena of suddenly expanding flows are studied by many researchers in this field due to its importance as explained above. Passive control is used often to control the base pressure whereas active control is rarely used. Literature works pertinent to suddenly expanded flows are reviewed as follows. Korst [6] studied the problem of base pressure problem in the regime of transonic as well as supersonic flow where the oncoming flow at the base was sonic and also supersonic after the wake. Depending upon the interaction among the adjacent free stream and the dissipative shear flow considering the mass in the wake,

Revised Manuscript Received on July 10,2019

Fharukh Ahmed G. M. Reaersch Scholar, Department of Mechanical Engineering, Bearys Institute of Technology, Mangalore, Karnataka India (fharukh@gmail.com)

Sher Afghan Khan Department of Mechanical Engineering, Bearys Institute of Technology, Mangalore, India. (sakhan06@gmail.com)
Korst devised a model (physical). Anderson and Williams [7] studied the sudden

expansion of air by analyzing noise produced and base pressure in a cylindrical duct. The base pressure obtained was minimum due to the attached flow depending upon the duct to area ratio of the nozzle. Bar-Haim and Weihs [2] investigated the control of the boundary layer by reducing drag in fully submerged bodies. By the suction from the boundary layer, the pressure can be increased from the axisymmetric bodies leading to control separation and delaying transition.

Rathakrishnan and Sreekanth [8] analyzed flows with sudden enlargement in a pipe. The analyses revealed that the dimensionless base pressure strongly depends upon the ratio of overall pressure, area ratio, expansion ratio, and the ratio of length-to-diameter of the duct. It was shown in the study that for a particular area ratio and the ratio of overall pressure, an optimal ratio of length-to-diameter could be found which could result in total pressure (maximum) at the exit of the nozzle at the axis-symmetry line and minimum base pressure near the sudden enlargement plane. The reattachment and separation looked to strongly depend upon the area ratio on the inlet to enlargement.

Quadros et al. [9] used the L9 orthogonal array for the planning of experiments and found that the lower area ratios are useful in control than the higher area ratios. Analysis of variance and multiple linear regression analysis were performed for the obtained experimental results. Fifteen random test results were used for the prediction of accuracy to test two linear regression models. Their analysis revealed that the linear regression model is enough to predict the base pressure accurately with and without control. Computational fluid dynamics analysis were performed for further in-depth analysis and validation was performed with the experimental results. For Mach number 1.25 and nozzle diameter ratio of 1.6 experiments were performed for under-expanded and correctly expanded cases by Khan et al. [10], [11], [12], [21], [13]-[20]. Surprisingly, the flow filed became oscillatory for a particular combination of NPR and L/D ratio. They observed in the case of both controls and without control. Several other relevant research works carried out using active control include [17], [18], [22]-[29]. With this motivation, this article reports the change in base pressure in a sudden expanded converging-diverging nozzle flow with Mach number of 1.7, 2.3, and 2.7, the area ratio of 2.56, 5.06, and 7.56. The use of active control in the improvement 
of base pressure is studied thoroughly, and hence an understanding of the role of microjets is explored in this work.

\section{EXPERIMENTAL PROCEDURE}

Fig. 1 shows the essential features of sudden expansion flow filed is illustrated showing the reattachment point, expansion waves, and recirculation zone. The same concept is used to perform the experimental investigation with the application of four micro jets at the base as shown in Fig. 2. The experimental facility available at High-Speed Aerodynamics Laboratory (HSAL), IIT, Kanpur, is employed for the analysis. The experimental setup is schematically shown in Figure 2. The side view shown at the right side of Fig. 2 shows the presence of eight holes along the circular position outer to the nozzle exit. The holes marked with 'c' are the microjets placed suitably for blowing, and holes ' $\mathrm{m}$ ' marked in the Figure are to measure the base pressure $\left(P_{b}\right)$. By blowing air, active control is accomplished through the holes ' $c$ ' consuming the pressure from a tube connected through the blowing chamber as shown in Fig. 2. The blowing chamber uses the same pressure from the settling chamber.

The static wall pressure was recorded to assess the nature of the flow field in the duct (enlarged one). To accomplish the measurement of static pressure in the downstream of the duct the pressure taps were used. The measurement location was selected at a distance of $8 \mathrm{~mm}$ each, holes of $1.5 \mathrm{~mm}$ are made which are nine in number, and the remaining holes are prepared at a distance of $10 \mathrm{~mm}$. The Length to diameter ratio (L/D) used in this study is varied from 10 to 1 , and the readings for each ratio is recorded. The experiment was repeated for Mach numbers in the range from 1.7, 2.3, and 2.7. In literature usually, L/D ratio employed is 3 to 5 for without control cases. With control, this ratio can be different and are varied from 10 to 1 .

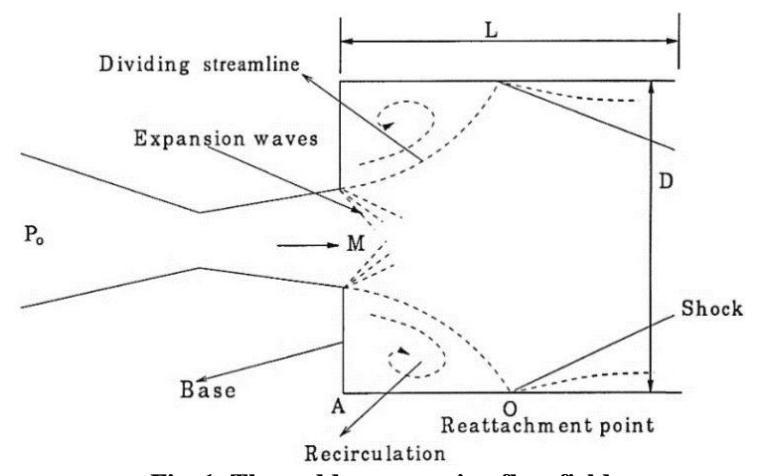

Fig. 1. The sudden expansion flow field

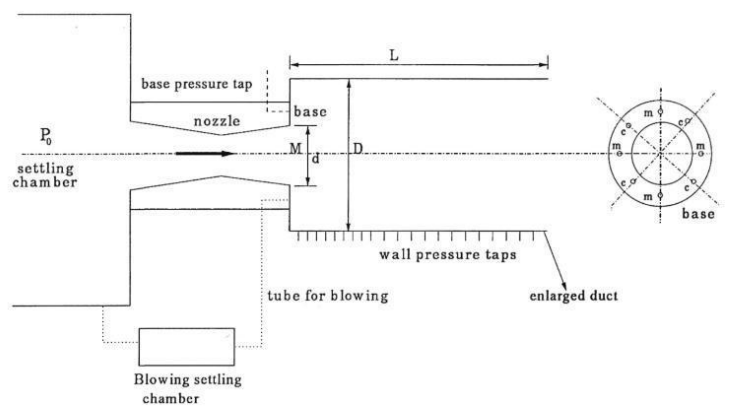

Fig. 2. Experimental setup for active control
For each value of each Mach number, and L/D ratio, with and without control. The NPR was varied from 1 to 10 in a step of 1 each, and the readings are noted for every single time. PSI System 2000 is used as a pressure transducer to record the change in base pressure variation. The pressure range is $0-300$ psi of the transducer employed, and it has sixteen channels. The sampling rate of the pressure transducer is two hundred fifty trials per second, and then the reading is exhibited on the monitor and recorded. The wall pressure was recorded using mercury manometer.

\section{RESULTS AND DISCUSSIONS}

This section devoted for discussion of results obtained from the experimental data, explains the effect of Mach number, NPR, area ratio, and L/D ratio on variation in base pressure in terms of \%change. The $\mathrm{L} / \mathrm{D}$ ratio which is the ratio of the length of the duct where the nozzle flow expands to the diameter of the duct. The area ratio is the ratio among the area of duct and area of the nozzle exit.

In Fig. 3 the \% change obtained in base pressure for Mach $1.7,2.3$, and 2.7 at L/D ratio at 10 with NPR increasing from 1 to 10 is shown. Figure 3-6 shows the \% change plots for Mach 1.7, 2.3, and 2.7 at L/D 10, 6, 4, and 2 respectively for a constant area ratio of 2.56 . The $\%$ change plotted is with respect to base pressure having no control. At Mach 1.7 the base pressure is increasing with a change in NPR. \% change of maximum 10 in base pressure is obtained at Mach 1.7 and NPR 10. At the nozzle exit, the flow gets separated at attaches itself with the duct wall at some distance from the exit similar to backward facing step. The area from the nozzle exit and reattachment forms a recirculation zone which contributes to a reduction in base pressure increasing the base drag. In this investigation, the orifice placed at the base of nozzle exit actively inject air which affects this recirculation zone. The NPR for correct expansion at Mach 1.7, 2.3, and 2.7 are 4.94, 12.5, and 23.3 respectively. At Mach 1.7, the flow undergoes under expansion below NPR of 4.94 and correct expansion at 4.94 and above this it undergoes overexpansion. For Mach 2.3 and 2.7, the flow remains under expanded at all NPR.The level of expansion $\left(P_{e} / P_{a}\right)$ for Mach 1.7 and NPR $=10$ is 2.03. Similarly, the level of over-expansion at the highest NPR for Mach numbers 2.3 and 2.7 is 0.8 and 0.43 (i.e., $P_{e} / P_{a}=0.8$, and 0.43$)$ respectively. From Fig. 3 to observe is the change at Mach 1.7 with NPR from 1 to 10 . At NPR 1 the control effectiveness is slight and then at NPR 3 it reversed and from NPR 4 to 10 continuous increase in $\%$ base is obtained. As high as a $15 \%$ increase in base pressure is recorded with active control. This increase has resulted from the jet placed at the dead zone where recirculation occurs leading to high base drag. The active control reduces this base drag by increasing the base pressure with an increase in NPR as the adverse pressure gradient keeps reducing and the expansion level increases. As the adverse gradient pressure completely vanishes, the control effectiveness increases. Coming to Mach 2.3, the base pressure reverses at NPR 5 and gains support from the control jets and nearly equals the amount of $\%$ increase at $\mathrm{NPR}=10$. 
At Mach 2.7, the control effectiveness has increased base pressure at all NPR. No adverse effect at Mach 2.7 for different NPR from 1 to 10 is seen. The maximum improvement in base pressure at highest NPR remained lower at Mach 2.7 then at Mach 1.7 and 2.3. At NPR 6 and Mach 2.7, the control has a lesser effect, and the base pressure is reduced but not has a negative impact at any NPR which is found in 1.7 and 2.3 Mach number. This concludes that at higher Mach numbers and NPR greater than 5 , the control is more effective in increasing the base pressure. The control breaks the recirculating zone increasing the base pressure suddenly to higher and higher values with increase in NPR values as shown in Fig. 3.

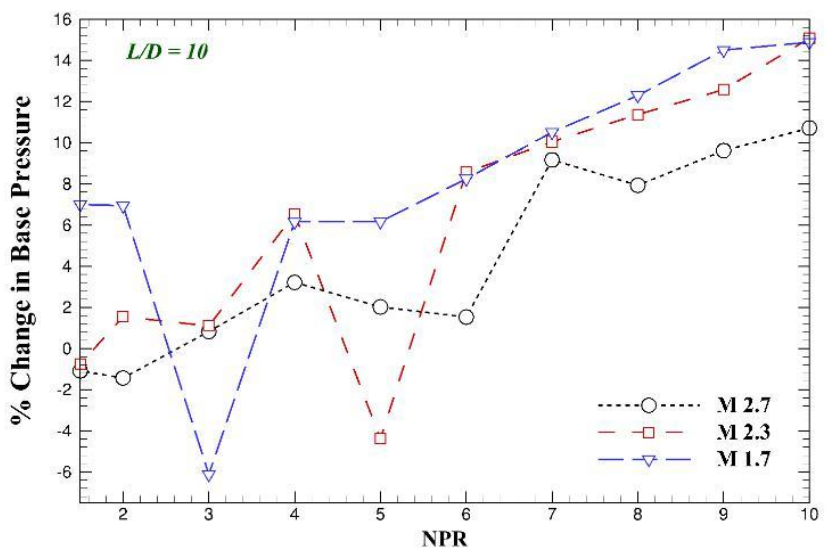

Fig. 3. Effect of NPR on base pressure at $L / D=10$

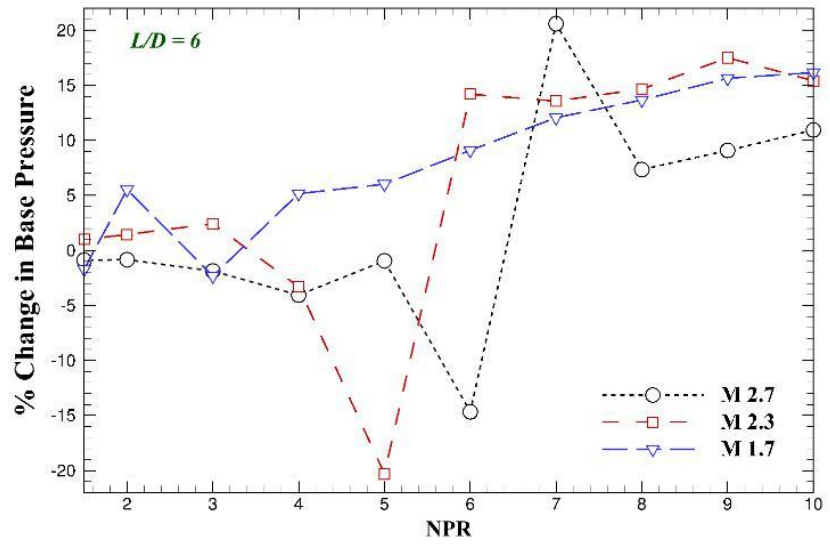

Fig. 4. Effect of NPR on base pressure at $L / D=6$

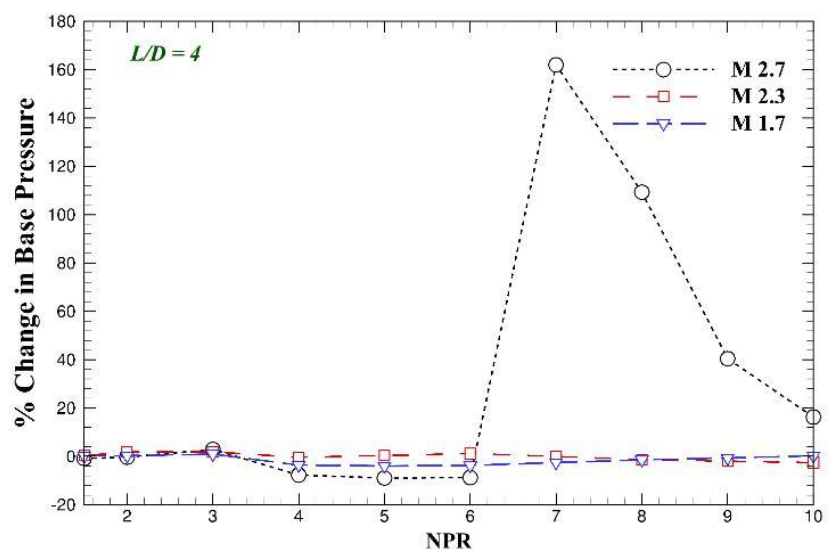

Fig. 5. Effect of $N P R$ on base pressure at $L / D=4$
Fig. 4-6 represent the change in base pressure at different L/D ratio and NPR from 1 to 10 . In Fig. 4 nature is nearly similar to the $\%$ change obtained in Fig. 3. With L/D 6, the dip in the base is obtained at NPR 3, 5, and 6 and remaining trend being same. To notice is the, a higher amount of increase in the base pressure at L/D 6 than at L/D 10. NPR 7 gives around $20 \%$ increase in base pressure than at other NPR. Similar results are seen in Fig. 5 shows a $160 \%$ increase in base pressure at NPR $=7$ and Mach 2.7. The different behavior of change in the base is obtained at $\mathrm{L} / \mathrm{D}=$ 2 at all NPRs. Almost at all NPR and Mach 1.7 and 2.the 3, the trend remains flat showing no effectiveness of ca control at $\mathrm{L} / \mathrm{D}=4$. Fig. 6 shows the enhancement with $\mathrm{L} / \mathrm{D}=2$ and area ratio of 2.56. As high as a $360 \%$ increase in dimensionless base pressure has resulted at NPR 6 and Mach 2.3. And at $L / D=2$ and NPR $=10$ for Mach 2.7, the base pressure has dropped to $-50 \%$ showing the negative impact of control on the pressure at the recirculation zone.

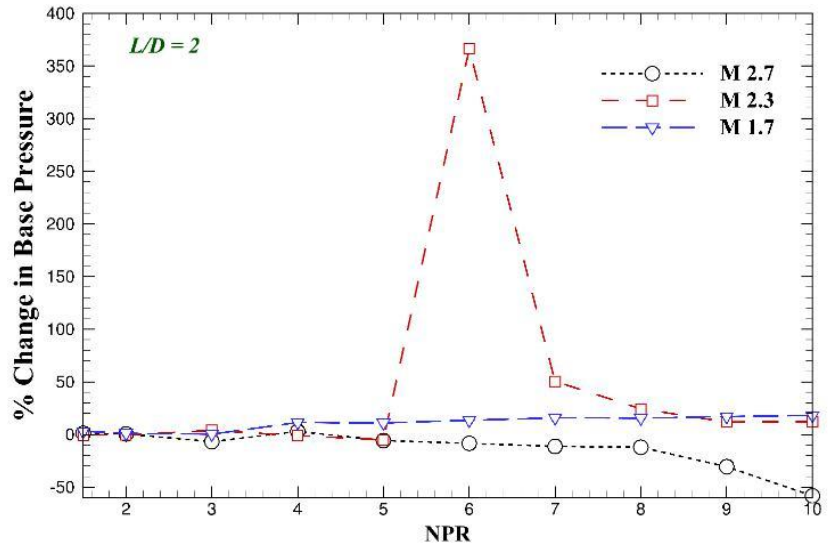

Fig. 6. Effect of NPR on base pressure at $L / D=2$

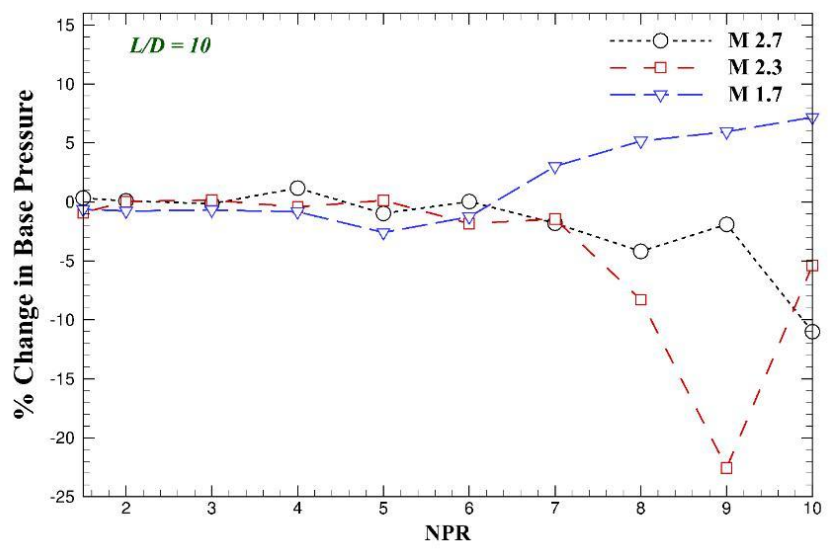

Fig. 7. Effect of NPR on base pressure at $L / D=10$

The physics behind this reveals that the reattachment zone being farther at Mach 2.7 the dead zone is entirely independent of this smaller $\mathrm{L} / \mathrm{D}$ ratio. The reattachment point is not obtained, and hence recirculation is not possible. With this, the control further disturbs the field and hence leads to a negative impact. Similar negative impacts at lower L/D ratio can be noticed ahead in Fig. 10 and Fig. 14. In this 
part, the effect of L/D and NPR is highlighted. But after this the effect of area ratio, i.e., the ratio of duct cross-section area and nozzle exit area is studied. At Mach 1.7 no effect of NPR from 1 to 10 at L/D $=2$ is due to the reduced length compared to its diameter.

In Fig. 7-10, the base pressure affected by the control at different NPR's, L/D ratio and area ratio of 5.06 (fixed) is shown. In Fig. 7 at NPR 1 to 7 the base pressure is unvaried by the control effectiveness, and for remaining NPR, the Mach number has a different effect. Mach 1.7 at this higher area ratio has a positive role combined with active control in increasing base pressure up to $10 \%$.

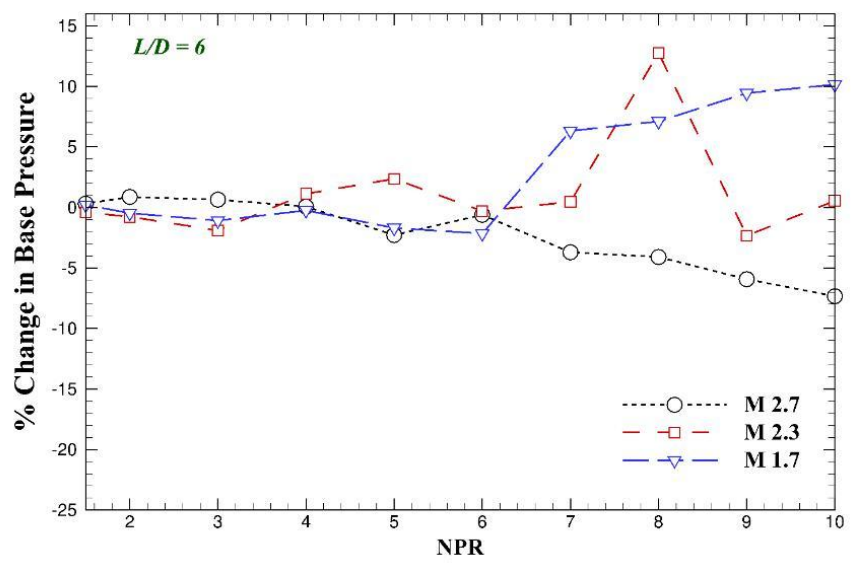

Fig. 8. Effect of NPR on base pressure at $L / D=6$

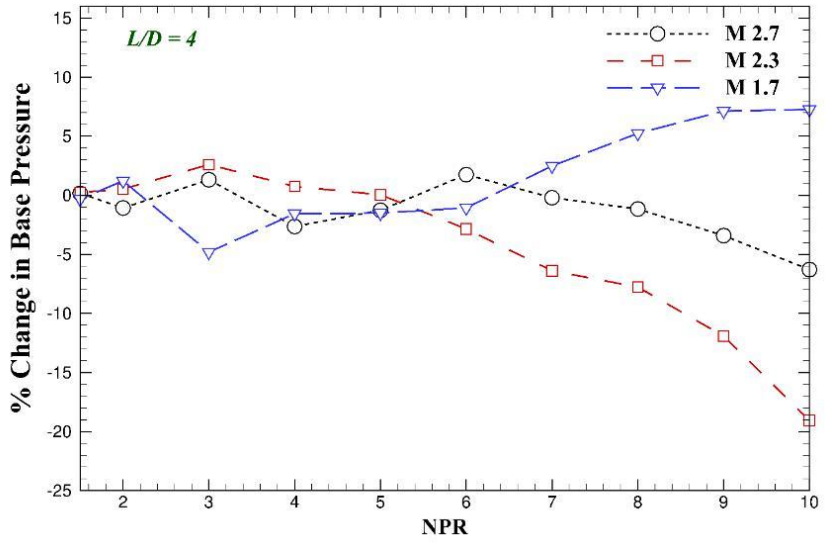

Fig. 9. Effect of NPR on base pressure at $L / D=4$

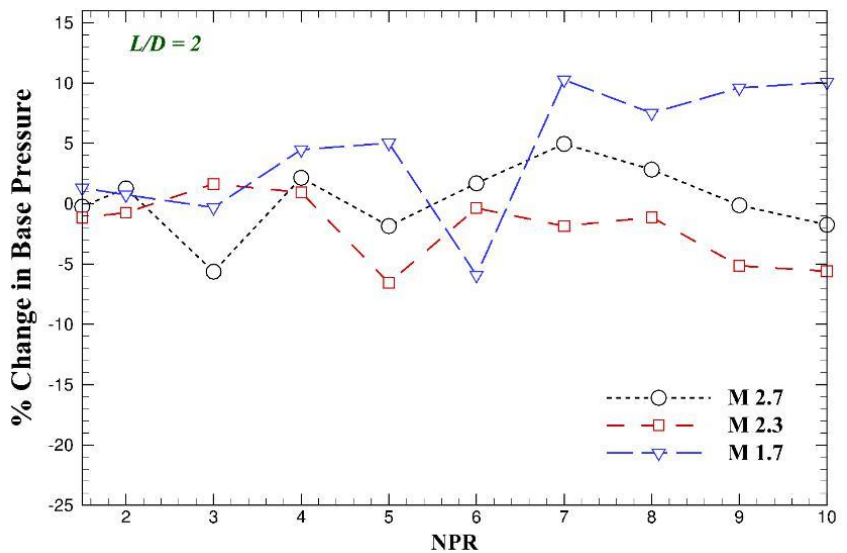

Fig. 10. Effect of NPR on base pressure at $L / D=2$
At Mach 2.3 and 2.7, the control has reversed the impact on base pressure due to increases in reattachment length with higher Mach number. For Mach 1.7 at L/D $=6$, the values are nearly the same. For Mach 2.3 the reversed effect is avoided with a reduction in length of the duct. The effect on Mach 2.7 seems to be not much altered for all NPR range. In Fig. 9 the effect on base pressure is very close to the effect obtained in Fig. 7. Mach 1.7 provides positive results while Mach 2.3 and 2.7 give negative base pressure implying the reversed effect of control on base pressure.

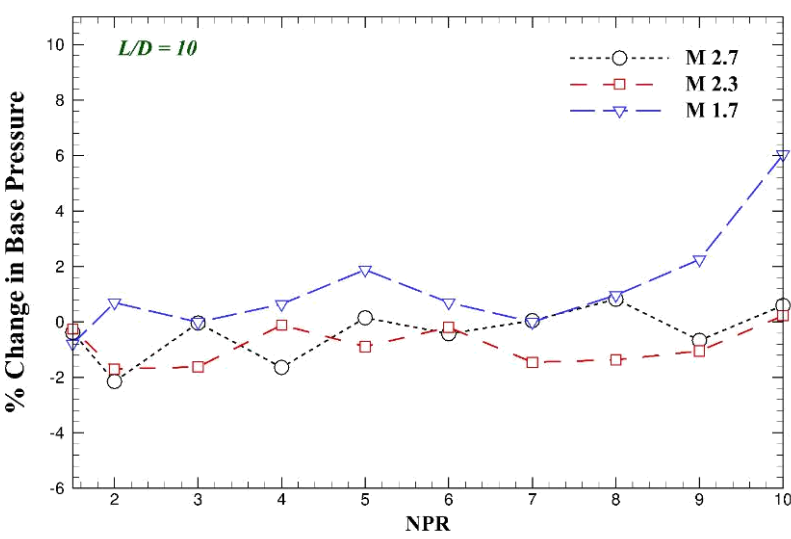

Fig. 11. Effect of NPR on base pressure at $L / D=10$

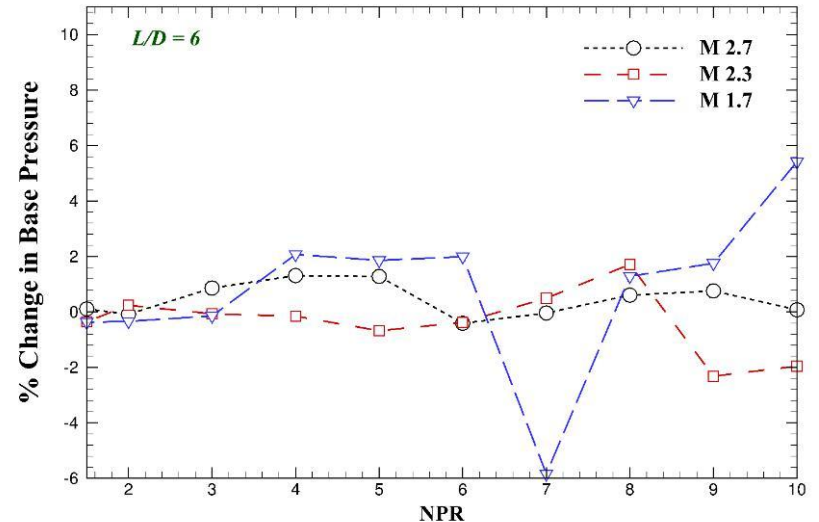

Fig. 12. Effect of NPR on base pressure at $L / D=6$

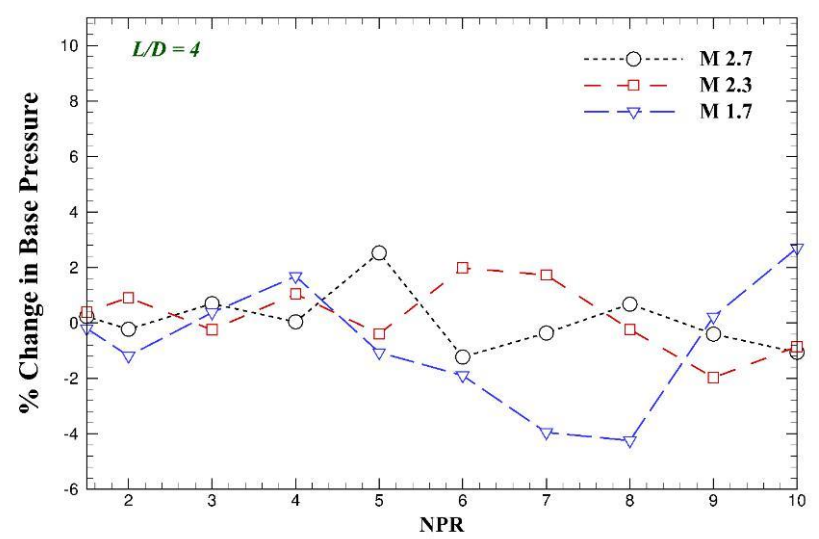

Fig. 13. Effect of NPR on base pressure at $L / D=4$ 


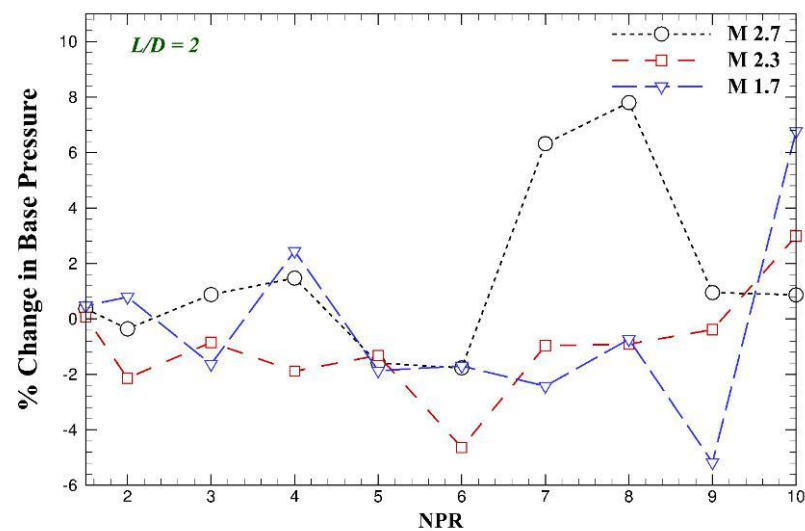

Fig. 14. Effect of NPR on base pressure at $L / D=2$

The \%change with an increase in NPR is shown in Fig. 10 at $\mathrm{L} / \mathrm{D}=2$ for Mach 1.7, 2.3, and 2.7 and area ratio of 5.06. At $L / D=2$ the variations in base pressure are very prominent at all NPRs. The prominent feature associated with the lower length of the duct is the dissociation of fluid from the duct wall. If no attachment is established, it leads to no circulation of the fluid in the dead zone at the base of the nozzle exit.

This impacts severely the base pressure as well as the role of the tiny jets in reducing the base drag. The change in base pressure at $\mathrm{L} / \mathrm{D}=10,6,4$, and 2 at the area of 7.56 and Mach 1.7, 2.3, and 2.7 is shown in Fig. 11-14 respectively. The changes are familiar with preceding results, and only at lower $\mathrm{L} / \mathrm{D}$ value, the active effect is noticeable.

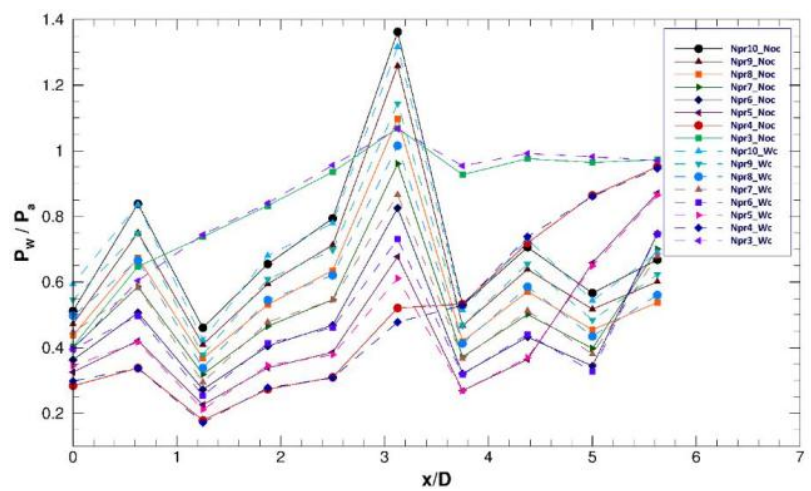

a) 1.7

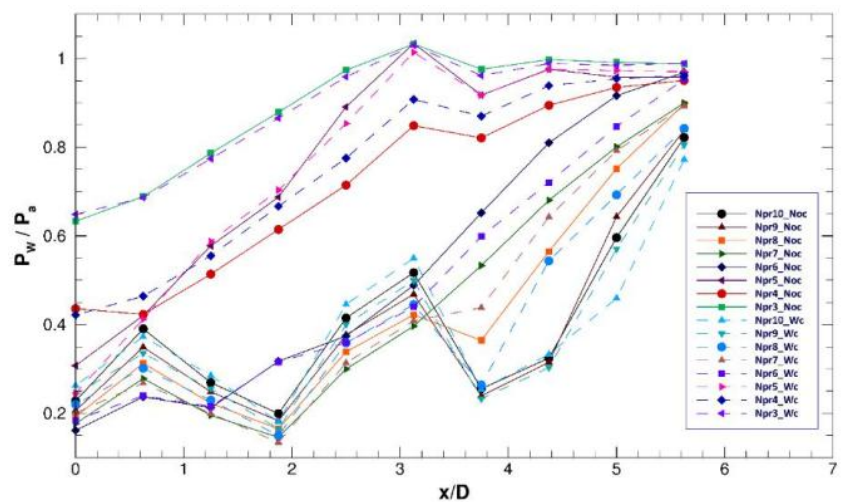

b) 2.3

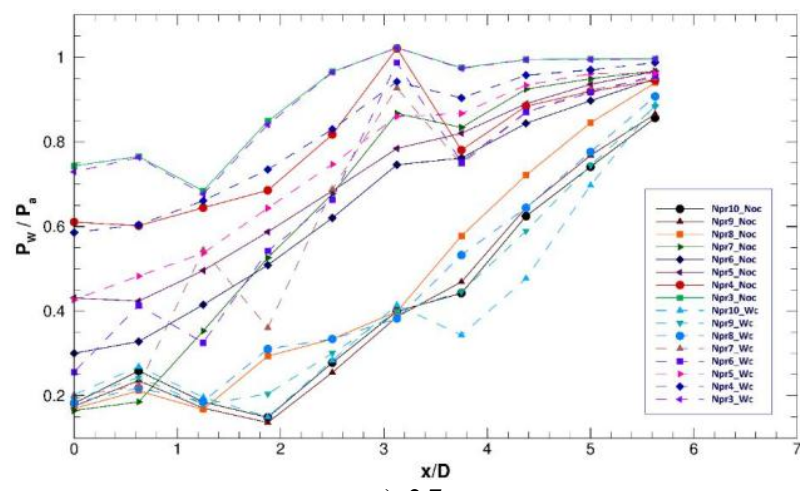

c) 2.7

Fig. 15. Wall pressure for Mach 1.7, 2.3, and 2.7 at $\mathrm{L} / \mathrm{D}=6$

The distribution of static wall pressure for Mach 1.7, 2.2, and last 2.7 are shown in the below Fig. 15 considering fixed $L / D=6$ for different NPR. The pressure field seems to behave identically with control and no control. Hence the wall pressure does not get influenced adversely leading further to oscillate violently due to active control. Whenever we employ the control using either active or passive control in increasing the base pressure; the primary issue associated with control of base flow related to wall pressure in supplementing the oscillatory nature is completely evaded.

\section{CONCLUSION}

In this article, the role of active control using jets of 1 diameter is analyzed on the occasion of suddenly expanded flows. The analysis is reported in the form of the amount of change in base pressure at the nozzle exit. The \%change obtained in base pressure for Mach 1.7, 2.3, and 2.7 belonging to the supersonic flow are studied. The length of the duct where the flow expands is varied from 10, 6, 4, and 2. The area ratio of duct and nozzle exit is chosen as 2.56 , 5.06, and 7.56. The results show that at higher Mach numbers and NPR greater than 5, the control is more effective in increasing the base pressure at 2.56 of area ratio and $L / D=10$. The area ratio of 5.06 and 7.56 are found to be less suitable with active control as most of the times the pressure remains unaltered. The wall pressure distribution, however, suggests that with the employment of active control the flow filed remains unaltered.

\section{REFERENCES}

1. A. K. Rathakrishnan, E, Sreekanth, "Flows in pipes with sudden enlargement," in 14th International Symposium on Space Technology and Science, 1984, pp. 491-496.

2. P. R. Viswanath, "Passive Devices for Axisymmetric Base Drag Reduction at Transonic Speeds," J. Aircr., vol. 25, no. 3, pp. 258-262, 1988.

3. E. Rathakrishnan, O. V Ramanaraju, and K Padmanabhan, "Influence of Cavities on Suddenly Expanded Flow Field," Mech. Res. Commun., vol. 16, no. 3, pp. 139-146, 1989.

4. E. Rathakrishnan, "Effect of Ribs on Suddenly Expanded Flows," AIAA J., vol. 39, no. 7, pp. 1402-1404, 2001.

5. R. Srikanth and E. Rathakrishnan, "Flow through pipes with sudden enlargement," Mech. Res. Commun., vol. 18, no. 4, pp. 199-206, 1991.

Published By: 
6. H. Korst, "A theory of base pressure in transonic and supersonic flow," J. Appl. Mech., vol. 23, no. 4, pp. 593-600, 1956.

7. J. S. Anderson and T. J. Williams, "Base pressure and noise produced by the abrupt expansion of air in a cylindrical duct," $J$. Mech. Eng. Sci., vol. 10, no. 3, pp. 262-268, 1968

8. S. A. Khan and E. Rathakrishnan, "Active Control of Suddenly Expanded Flows from Overexpanded Nozzles," Int. J. Turbo Jet Engines, vol. 19, pp. 119-126, 2002.

9. J. D. Quadros, S. A. Khan, and A. J. Antony, "Effect of Flow Parameters on Base Pressure in a Suddenly Expanded Duct at Supersonic Mach number Regimes using CFD and Design of Experiments," J. Appl. Fluid Mech., vol. 11, no. 2, pp. 483-496, 2016.

10. S. A. Khan, M. Bashir, F. A. G. M, and M. A. Ullah, "An investigation of base flow control by wall pressure analysis in a sudden expansion nozzle," J. Sci. Res. Dev., vol. 3, no. 5, pp. 16, 2016.

11. S. A. Khan and E. Rathakrishnan, "Control of Suddenly Expanded Flows from Correctly Expanded Nozzles," Int. J. Turbo Jet Engines, vol. 21, pp. 255-278, 2004.

12. S. A. Khan and E. Rathakrishnan, "Active Control of Suddenly Expanded Flows from Underexpanded Nozzles - Part II," Int. J. Turbo Jet Engines, vol. 22, pp. 163-183, 2005.

13. S. A. Khan and E. Rathakrishnan, "Nozzle Expansion Level Effect on Suddenly Expanded Flow," Int. J. Turbo Jet Engines, vol. 23, 233-257, 2006

14. S. A. Khan and E. Rathakrishnan, "Active Control of Base Pressure in Supersonic Regime," J. Inst. Eng. Aerosp. Eng. J., vol. 87, pp. 1-10, 2006

15. S. Rehman and S. A. Khan, "Control of base pressure with microjets : part I," Aircr. Eng. Aerosp. Technol. An Int. J., vol. 80, no. 2, 158-164, 2008

16. S. A. Khan, M. Asadullah, F. A. G. M, A. Jalaluddeen, and A. Ahmed, Maughal Baig, "Flow Control with Aerospike Behind Bluff Body," Int. J. Mech. Prod., vol. 8, no. 3, pp. 1001-1008, 2018.

17. S. A. Khan, M. Asadullah, F. A. G. M, A. Jalaluddeen, and A. Ahmed, Maughal Baig, "Passive Control of Base Drag in Compressible Subsonic Flow Using Multiple Cavity," Int. J. Mech. Prod., vol. 8, no. 4, pp. 39-44, 2018.

18. S. A. Khan, M. Asadullah, and J. Sadhiq, "Passive Control of Base Drag Employing Dimple in Subsonic Suddenly Expanded Flow," Int. J. Mech. Mechatronics Eng., vol. 18, no. 3, pp. 69-74, 2018

19. S. A. Khan and E. Rathakrishnan, "Control of suddenly expanded flow," Aircr. Eng. Aerosp. Technol. An Int. J., vol. 78 , no. 4, pp. 293-309, 2006.

20. S. A. Khan and E. Rathakrishnan, "Active Control of Suddenly Expanded Flows from Underexpanded Nozzles," Int. J. Turbo Jet Engines, vol. 21, pp. 233-254, 2004

21. S. A. Khan and E. Rathakrishnan, "Control of Suddenly Expanded Flows with Micro-Jets," Int. J. Turbo Jet Engines, vol. 20, pp. 63-81, 2003.

22. F. A. G. M, M. A. Ullah, and S. A. Khan, "Experimental Study of Suddenly Expanded Flow from Nozzle," ARPN J. Eng. Appl. Sci., vol. 11, no. 16, pp. 10041-10047, 2016.

23. J. Quadros, S. A. Khan, and A J Antony, "Study of Effect of Flow Parameters on Base Pressure in a Suddenly Expanded Duct at Supersonic Mach Number Regimes using CFD and Design of Experiments," J. Appl. Fluid Mech., vol. 11, no. 2, pp. 483-496 2018.

24. S. A. Khan, Z. I. Chaudhary, and V. B. Shinde, "Base Pressure Control by Supersonic Micro Jets in a Suddenly Expanded Nozzle," Int. J. Mech. Mechatronics Eng., vol. 18, no. 4, pp. $101-113,2018$.

25. F. Ahmed and S. A. Khan, "Investigation of the efficacy of low length-to-diameter ratio and nozzle pressure ratio on base pressure in an abruptly expanded flow," in MATEC Web of Conferences, 2018, vol. 01004, pp. 1-6.

26. M. Asadullah, S. A. Khan, W. Asrar, and E. Sulaeman, "Passive control of base pressure with the static cylinder at supersonic flow," Int. Conf. Aerosp. Mech. Eng., vol. 370, p. 012050, 2018.

27. M. Asadullah, S. A. Khan, W. Asrar, and E. Sulaeman, "Low-Cost Base Drag Reduction Technique," Int. J. Mech. Eng. Robot. Res., vol. 7, no. 4, pp. 428-432, 2018.

28. A. A. Alrobaian, S. A. Khan, M. Asadullah, F. A. G. M, and Imtiyaz A, "A new approach to the low-cost open-typed subsonic compressible flow wind tunnel for academic purpose," Int. J. Mech. Prod., vol. 8, no. 6, pp. 383-394, 2018

29. N. S. Vikramaditya, M. Viji, S. B. Verma, N. Ali, and D. N. Thakur, "Base Pressure Fluctuations on Typical Missile
Configuration in the presence of Base Cavity," J. Spacecr. Rockets, vol. 55, no. 2, pp. 335-345, 2018. 